\title{
Relativistic Brueckner-Hartree-Fock theory for neutron drops
}

\author{
Shihang Shen (申时行), ${ }^{1,2}$ Haozhao Liang (梁豪兆), ${ }^{3,4}$ Jie Meng (孟杰) ${ }^{1,5,6,{ }^{*}}$ Peter Ring, ${ }^{1,7}$ and Shuangquan Zhang (张双全) ${ }^{1}$ \\ ${ }^{1}$ State Key Laboratory of Nuclear Physics and Technology, School of Physics, Peking University, Beijing 100871, China \\ ${ }^{2}$ Department of Physics, The University of Hong Kong, Pokfulam Road, Hong Kong, China \\ ${ }^{3}$ RIKEN Nishina Center, Wako 351-0198, Japan \\ ${ }^{4}$ Department of Physics, Graduate School of Science, The University of Tokyo, Tokyo 113-0033, Japan \\ ${ }^{5}$ Department of Physics, University of Stellenbosch, Stellenbosch, South Africa \\ ${ }^{6}$ Yukawa Institute for Theoretical Physics, Kyoto University, Kyoto 606-8502, Japan \\ ${ }^{7}$ Physik-Department der Technischen Universität München, D-85748 Garching, Germany
}

(Received 24 February 2018; published 11 May 2018)

\begin{abstract}
Neutron drops confined in an external field are studied in the framework of relativistic Brueckner-Hartree-Fock theory using the bare nucleon-nucleon interaction. The ground-state energies and radii of neutron drops with even numbers from $N=4$ to $N=50$ are calculated and compared with results obtained from other nonrelativistic $a b$ initio calculations and from relativistic density functional theory. Special attention has been paid to the magic numbers and to the subshell closures. The single-particle energies are investigated and the monopole effect of the tensor force on the evolutions of the spin-orbit and the pseudospin-orbit splittings is discussed. The results provide interesting insights into neutron-rich systems and can form an important guide for future density functionals.
\end{abstract}

DOI: 10.1103/PhysRevC.97.054312

\section{INTRODUCTION}

The shell structure in atomic nuclei is one of the most astonishing facts. It was discovered in the late 1940s [1,2] and forms the foundation of nuclear physics since then. With the advance of radioactive-ion-beam facilities around the world, more and more neutron-rich exotic nuclei have been reached, where many interesting new phenomena emerge such as the disappearance of traditional and the appearance of new magic numbers [3-5], and the halo phenomena [6-8]. These new findings present challenges for current nuclear structure theory, in particular for nuclear density functional theory which should provide a uniform description over the entire nuclear chart [9-13]. In this framework there is no possibility to adjust the effective interaction or the resulting single-particle energies separately for each area of the chart, as it is done in many shell-model configuration-interaction (CI) calculations. Nuclear density functionals, even though they are phenomenological, are usually obtained by fitting to the properties of stable nuclei and, therefore, they are not well constrained in exotic regions far from the line of $\beta$ stability. Microscopic calculations started from nucleonnucleon $(N N)$ interactions, or the so-called ab initio calculations [14-23], can provide valuable information to understand nuclear structure but are still difficult to apply to exotic nuclei.

The neutron drop provides an ideal simple model to investigate the neutron-rich environment. It is composed of a finite number of pure neutrons which are constrained in an external field to keep the neutrons bound. Because only the

*mengj@pku.edu.cn neutron-neutron interaction exists, equations for neutron drops are much easier to solve. Therefore they can be calculated by many ab initio methods [24-28]. In this way, different methods and different interactions can be compared, and valuable information can be obtained for constraining nuclear density functionals in neutron-rich system.

The neutron drops were first studied by quantum Monte Carlo methods [24] for $N=7$ and 8 using the two-nucleon $(2 N)$ interaction Argonne $v_{18}[29]$ and the three-nucleon $(3 N)$ interaction Urbana IX [30]. It was found that commonly used Skyrme functionals overestimate the central density of these drops and the spin-orbit splitting of drops with seven neutrons [24]. In Ref. [31] the ground-state energy was studied for $N=6$ neutron drops and the neutron pairing energy was discussed by comparison with Ref. [24]. Later, more systematic studies were performed for larger $N$ values with different external fields and different interactions by using quantum Monte Carlo methods [26,32-34]. Studies with the modern high-precision chiral $2 N$ interaction $\mathrm{N}^{3} \mathrm{LO}$ [35] and the $3 N$ interaction $\mathrm{N}^{2} \mathrm{LO}$ [36] have been benchmarked with different $a b$ initio methods, including the no-core shell model [18] and the coupled-cluster theory [19], and it was found that the results are consistent with each other [27]. However, by comparing these $a b$ initio calculations, one found a significant dependence on the selected interactions, especially on the $3 \mathrm{~N}$ interactions [26,27,34].

On the other hand, various nonrelativistic and relativistic density functionals have been used to study neutron drops, and a strong linear correlation between the rms radii of neutron drops and the neutron skin thickness of ${ }^{208} \mathrm{~Pb}$ and ${ }^{48} \mathrm{Ca}$ has been pointed out in Ref. [37]. Because of the uncertainty in the isovector part, there exists a large uncertainty in the results of neutron drops for the different functionals. 
Recently, the self-consistent relativistic Brueckner-HartreeFock (RBHF) theory for finite nuclei has been established, and the results are in much better agreement with experimental data than the nonrelativistic calculations with the $2 N$ interaction only [22,23]. Indeed, it has been known for over than 30 years that relativistic Brueckner-Hartree-Fock theory gives a much better description of the nuclear matter saturation properties than nonrelativistic BHF theories [38-40]. In nonrelativistic many-body investigations on the influence of various types of $3 N$ interactions, it was found that a relativistic effect, the so-called $Z$ diagram, plays a major role [41].

With progresses in mind, it is important to study the neutron drops in more detail in the framework of RBHF theory and compare the results with those of other nonrelativistic ab initio calculations using various $2 N$ or $2 N+3 N$ interactions, as well as calculations using various density functionals. This can also provide valuable insight to improve current relativistic density functionals. In Ref. [28], a systematic and specific pattern due to the tensor forces in the evolution of spin-orbit splittings based on RBHF theory is reported.

In this work, we investigate neutron drops confined in an external harmonic-oscillator potential using relativistic Brueckner-Hartree-Fock theory and present the numerical details and calculated results in detail. In Sec. II, we give a brief outline of the RBHF framework for neutron drops. The numerical details are discussed in Sec. III. Results and discussion for neutron drops with an even number of neutrons from $N=4$ to 50 are presented in Sec. IV. Finally, a summary and perspectives for future investigations is given in Sec. V.

\section{THEORETICAL FRAMEWORK}

In this section, we outline the theoretical framework of relativistic Brueckner-Hartree-Fock theory for neutron drops. For a detailed description of RBHF theory for finite nuclei, we refer the reader to Refs. [22,23].

We start with a relativistic one-boson-exchange $N N$ interaction which describes the $N N$ scattering data [42]:

$$
\begin{aligned}
\mathscr{L}_{N N p v} & =-\frac{f_{p s}}{m_{p s}} \bar{\psi} \gamma^{5} \gamma^{\mu} \psi \partial_{\mu} \varphi^{(p s)}, \\
\mathscr{L}_{N N s} & =g_{s} \bar{\psi} \psi \varphi^{(s)}, \\
\mathscr{L}_{N N v} & =-g_{v} \bar{\psi} \gamma^{\mu} \psi \varphi_{\mu}^{(v)}-\frac{f_{v}}{4 M} \bar{\psi} \sigma^{\mu v} \psi\left(\partial_{\mu} \varphi_{v}^{(v)}-\partial_{\nu} \varphi_{\mu}^{(v)}\right),
\end{aligned}
$$

where $\psi$ denotes the nucleon field. The bosons to be exchanged are characterized by the index $\alpha$ and include the pseudoscalar mesons $\eta, \pi$ with a pseudovector $p v$ coupling, the scalar $(s)$ mesons $\sigma, \delta$, and the vector $(v)$ mesons $\omega, \rho$. For each pair, e.g., $\eta, \pi$, the first (second) meson has isoscalar (isovector) character. For the isovector mesons, the field operator $\varphi_{\alpha}$ is replaced by $\vec{\varphi}_{\alpha} \cdot \vec{\tau}$, with $\vec{\tau}$ being the usual Pauli matrices in isospace.

The Hamiltonian is obtained through the Legendre transformation. Considering the stationary case, the Hamiltonian can be expressed in the second-quantized form as

$$
H=\sum_{k k^{\prime}}\left\langle k|T| k^{\prime}\right\rangle b_{k}^{\dagger} b_{k^{\prime}}+\frac{1}{2} \sum_{k l k^{\prime} l^{\prime}}\left\langle k l|V| k^{\prime} l^{\prime}\right\rangle b_{k}^{\dagger} b_{l}^{\dagger} b_{l^{\prime}} b_{k^{\prime}},
$$

where the relativistic matrix elements are given by

$$
\begin{aligned}
\left\langle k|T| k^{\prime}\right\rangle= & \int d^{3} r \bar{\psi}_{k}(\mathbf{r})(-i \boldsymbol{\gamma} \cdot \nabla+M) \psi_{k^{\prime}}(\mathbf{r}), \\
\left\langle k l\left|V_{\alpha}\right| k^{\prime} l^{\prime}\right\rangle= & \int d^{3} r_{1} d^{3} r_{2} \bar{\psi}_{k}\left(\mathbf{r}_{1}\right) \Gamma_{\alpha}^{(1)} \psi_{k^{\prime}}\left(\mathbf{r}_{1}\right) \\
& \times D_{\alpha}\left(\mathbf{r}_{1}, \mathbf{r}_{2}\right) \bar{\psi}_{l}\left(\mathbf{r}_{2}\right) \Gamma_{\alpha}^{(2)} \psi_{l^{\prime}}\left(\mathbf{r}_{2}\right) .
\end{aligned}
$$

The indices $k, l$ run over an arbitrary complete basis of Dirac spinors with positive and negative energies, such as, for instance, over the eigensolutions of a Dirac equation with potentials of Woods-Saxon shape discussed in Refs. [23,43,44]. The two-body interaction $V$ contains contributions from the different mesons $\alpha$. The interaction vertices for particles 1 and 2 are $\Gamma_{\alpha}^{(1)}$ and $\Gamma_{\alpha}^{(2)}$ :

$$
\begin{aligned}
\Gamma_{s} & =g_{s}, \\
\Gamma_{p v} & =\frac{f_{p s}}{m_{p s}} \gamma^{5} \gamma^{i} \partial_{i}, \\
\Gamma_{v}^{\mu} & =g_{v} \gamma^{\mu}+\frac{f_{v}}{2 M} \sigma^{i \mu} \partial_{i} .
\end{aligned}
$$

In the Bonn interaction, there is a form factor of monopole type attached to each vertex. It has the following form in momentum space:

$$
\frac{\Lambda_{\alpha}^{2}-m_{\alpha}^{2}}{\Lambda_{\alpha}^{2}+\mathbf{q}^{2}},
$$

where $\Lambda_{\alpha}$ is the cutoff parameter for meson $\alpha$ and $\mathbf{q}$ is the momentum transfer following Ref. [42].

The meson propagators $D_{\alpha}\left(\mathbf{r}_{1}, \mathbf{r}_{2}\right)$ are the retarded solutions of the Klein-Gordon equations in Minkowski space. For the Bonn interaction, this retardation effect was deemed to be small and was ignored from the beginning [42]. In this way, the $q_{0}$ integration can be carried out and we are left with the meson propagators in $r$ space:

$$
D_{\alpha}\left(\mathbf{r}_{1}, \mathbf{r}_{2}\right)= \pm \int \frac{d^{3} q}{(2 \pi)^{3}} \frac{1}{m_{\alpha}^{2}+\mathbf{q}^{2}} e^{i \mathbf{q} \cdot\left(\mathbf{r}_{1}-\mathbf{r}_{2}\right)} .
$$

The negative sign holds for scalar (and pseudoscalar) mesons and the positive sign for the vector fields. Note that, with the form factor in Eq. (6), the meson propagators are no longer simple Yukawa functions, but they can be evaluated in analytic form [45].

The matrix elements of the bare nucleon-nucleon interaction are very large and difficult to be used directly in nuclear many-body theory. Within Brueckner theory, the bare interaction is replaced by an effective interaction in the nuclear medium: the $G$ matrix. It takes into account short-range correlations by summing up all the ladder diagrams of the bare interaction $[46,47]$ and is deduced from the Bethe-Goldstone 
(BG) equation [48],

$$
\begin{aligned}
\left\langle a b|\bar{G}(W)| a^{\prime} b^{\prime}\right\rangle= & \left\langle a b|\bar{V}| a^{\prime} b^{\prime}\right\rangle+\frac{1}{2} \sum_{c d}\langle a b|\bar{V}| c d\rangle \\
& \times \frac{Q(c, d)}{W-\varepsilon_{c}-\varepsilon_{d}}\left\langle c d|\bar{G}(W)| a^{\prime} b^{\prime}\right\rangle,
\end{aligned}
$$

where $|a\rangle,|b\rangle$ are solutions of the relativistic Hartree-Fock equations, $\left\langle a b|\bar{V}| a^{\prime} b^{\prime}\right\rangle=\left\langle a b|V| a^{\prime} b^{\prime}-b^{\prime} a^{\prime}\right\rangle$ are the antisymmetrized two-body matrix elements, $W$ is the starting energy, and $\varepsilon_{c}, \varepsilon_{d}$ are the single-particle energies of the two particles in the intermediate states. The Pauli operator $Q(c, d)$ allows scattering only to states $c$ and $d$ above the Fermi surface. We also do not allow scattering to states in the Dirac sea. Therefore, $Q(c, d)$ is defined as

$$
Q(c, d)= \begin{cases}1, & \text { for } \varepsilon_{\mathrm{c}}>\varepsilon_{\mathrm{F}} \text { and } \varepsilon_{\mathrm{d}}>\varepsilon_{\mathrm{F}} \\ 0, & \text { otherwise. }\end{cases}
$$

The single-particle motion fulfills the relativistic HartreeFock (RHF) equation, which in an external field reads

$$
\left(T+U+U_{\mathrm{ex}}\right)|a\rangle=e_{a}|a\rangle,
$$

where $e_{a}=\varepsilon_{a}+M$ is the single-particle energy with the rest mass of the nucleon $M$, and $U_{\mathrm{ex}}$ is the external field to confine the neutron drop. The self-consistent single-particle potential $U$ is defined with the $G$ matrix by $[49,50]$ :

$$
U_{a b}=\frac{1}{2} \sum_{c=1}^{N}\left\langle a c\left|\bar{G}\left(\varepsilon_{a}+\varepsilon_{c}\right)+\bar{G}\left(\varepsilon_{b}+\varepsilon_{c}\right)\right| b c\right\rangle,
$$

if $|a\rangle$ and $|b\rangle$ are both hole (i.e., occupied) states, and

$$
U_{a b}=\sum_{c=1}^{N}\left\langle a c\left|\bar{G}\left(\varepsilon_{a}+\varepsilon_{c}\right)\right| b c\right\rangle,
$$

if $|a\rangle$ is a hole state and $|b\rangle$ is a particle (i.e., unoccupied) state, and

$$
U_{a b}=\frac{1}{2} \sum_{c=1}^{N}\left\langle a c\left|\bar{G}\left(\varepsilon_{a}^{\prime}+\varepsilon_{c}\right)+\bar{G}\left(\varepsilon_{b}^{\prime}+\varepsilon_{c}\right)\right| b c\right\rangle,
$$

if $|a\rangle$ and $|b\rangle$ are both particle states. In the above expressions, the summation index $c$ goes through $N$ neutron-occupied states.

In the above expressions, $\varepsilon$ labels the self-consistent singleparticle energies, while $\varepsilon^{\prime}$ is somewhat uncertain [50]. The matrix elements of the self-consistent potential $U_{a b}$ with both states $|a\rangle$ and $|b\rangle$ above the Fermi level are not well defined in the Brueckner-Hartree-Fock theory. Different choices have been proposed in the literature [50,51]. Following the discussions in Ref. [23,50], we choose $\varepsilon_{a}^{\prime}=\varepsilon_{b}^{\prime}=\varepsilon_{1 s 1 / 2}$ fixed as the lowest energy of the occupied states in the Fermi sea.

\section{NUMERICAL DETAILS}

We use the Bonn interaction adjusted to the $N N$ scattering data in Ref. [42]. The neutron drops will be confined in a spherical harmonic oscillator (HO) trap, i.e., the external field in Eq. (10) is

$$
U_{\mathrm{ex}}=\frac{1}{2} M \omega^{2} r^{2}
$$

where the strength is chosen as $\hbar \omega=10 \mathrm{MeV}$ if without specification. In contrast to the relativistic Brueckner-HartreeFock calculations for self-bound nuclei in Refs. [22,23], where we had to introduce a center-of-mass correction, this is not necessary here because, in the external field, translational symmetry is lost. The initial basis is the Dirac Woods-Saxon (DWS) basis [43], and during the RBHF iteration it will be transformed to the self-consistent RHF basis as explained in Ref. [23]. The DWS basis is obtained by solving the spherical Dirac equation in a box with the box size $R_{\mathrm{box}}=8 \mathrm{fm}$ and a mesh size $d r=0.05 \mathrm{fm}$. The way to solve the BG equation (8) is the same as in Refs. [22,23], except that now only the isospin channel $T_{z}=1$ is included.

It is well known that the bare $N N$ interaction contains a repulsive core and a strong tensor part connecting the nucleons below the Fermi surface to the states with high momentum in the continuum. To take this coupling fully into account, one needs a relatively large basis space. The convergence in finite nuclei has been confirmed in Refs. [22,23], in which reasonable convergence is achieved near an energy cutoff $\varepsilon_{\text {cut }}=1.1 \mathrm{GeV}$. For the neutron drops, we carry out the same check.

Figure 1 shows the total energy divided by $\hbar \omega N^{4 / 3}$ of neutron drops with $N=8,20,28$, and 50 in a HO trap $(\hbar \omega=$ $10 \mathrm{MeV}$ ) as a function of the energy cutoff $\varepsilon_{\text {cut }}$ calculated by RBHF theory using the Bonn A interaction. The factor $\hbar \omega N^{4 / 3}$ is based on the consideration that in Thomas-Fermi approximation [52] the total energy for a noninteracting $N$ fermion system in a $\mathrm{HO}$ trap is given by

$$
E=\frac{3^{4 / 3}}{4} \hbar \omega N^{4 / 3} \approx 1.082 \hbar \omega N^{4 / 3} .
$$

In other words, all the energy below the line $E / \hbar \omega N^{4 / 3} \approx$ 1.082 corresponds to binding induced through the nuclear force. This intrinsic binding energy grows linearly with $\hbar \omega$. With increasing neutron number of the drops we observe a saturation but, in contrast to the nuclear case where the binding energy grows roughly with mass number $A$, here it grows for large $N \geqslant 20$ with $N^{4 / 3}$.

It can be seen from Fig. 1 that, first, the convergence with the energy cutoff does not depend on particle number. Second,

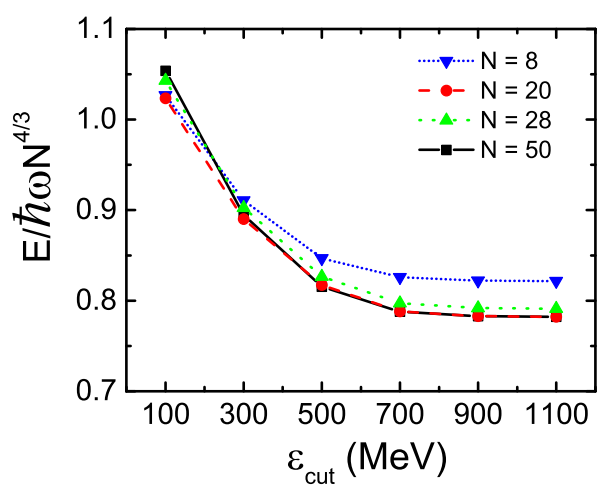

FIG. 1. Total energy (in units of $\hbar \omega N^{4 / 3}$ ) of neutron drops with $N=8,20,28$, and 50 in a $\mathrm{HO} \operatorname{trap}(\hbar \omega=10 \mathrm{MeV})$ as a function of the energy cutoff $\varepsilon_{\text {cut }}$ calculated by RBHF theory using the interaction Bonn A. 


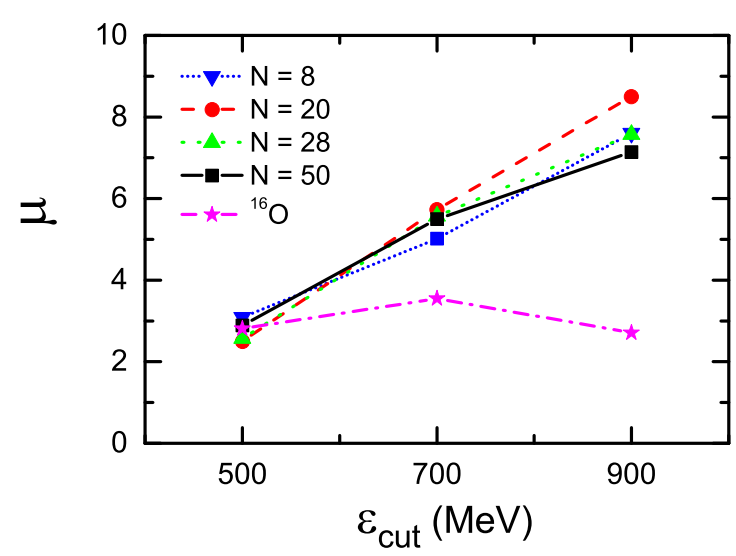

FIG. 2. Convergence rate $\mu$ for neutron drops with $N=8,20$, 28 , and 50 in a $\mathrm{HO}$ trap $(\hbar \omega=10 \mathrm{MeV})$ and for the nucleus ${ }^{16} \mathrm{O}$ as a function of the energy cutoff $\varepsilon_{\text {cut }}$ calculated by RBHF theory using the Bonn A interaction.

the convergence is achieved already at $\varepsilon_{\text {cut }}=900 \mathrm{MeV}$, which is faster than $\varepsilon_{\text {cut }}=1100 \mathrm{MeV}$ in finite nuclei [22,23]. This is because the tensor term plays a role in connecting the nucleons below the Fermi surface to the states with high momentum, and only the $T=1$ term shows in neutron drops.

To compare the speed of convergence between neutron drops and finite nuclei, from the total energy of the system we define the following convergence rate:

$$
\mu\left(\varepsilon_{\mathrm{cut}}\right)=\frac{E\left(\varepsilon_{\mathrm{cut}}-200 \mathrm{MeV}\right)-E\left(\varepsilon_{\mathrm{cut}}\right)}{E\left(\varepsilon_{\mathrm{cut}}\right)-E\left(\varepsilon_{\mathrm{cut}}+200 \mathrm{MeV}\right)} .
$$

The larger the quantity $\mu$ is, the faster the convergence is.

In Fig. 2, we show the convergence rate $\mu$ calculated with RBHF theory using the interaction Bonn A for neutron drops with $N=8,20,28$, and 50 in a $\mathrm{HO}$ trap with $\hbar \omega=10 \mathrm{MeV}$, and for the nucleus ${ }^{16} \mathrm{O}$ (from Refs. [22,23]). It can be seen that, at $\varepsilon_{\text {cut }}=500 \mathrm{MeV}$, the convergence rates between different neutron drops and ${ }^{16} \mathrm{O}$ are similar. As $\varepsilon_{\text {cut }}$ increases, the convergence rates of neutron drops increase linearly, and they are similar for neutron drops with different neutron numbers. On the other hand, the convergence rate of ${ }^{16} \mathrm{O}$ does not change too much as $\varepsilon_{\text {cut }}$ increases and it is much slower than that of neutron drops.

\section{RESULTS AND DISCUSSION}

\section{A. Total energy}

In Table I we list the total energies and root-mean-square (rms) radii of $N$-neutron drops in a $\mathrm{HO}$ trap $(\hbar \omega=10 \mathrm{MeV})$ calculated by RBHF theory using the interactions Bonn A, B, and $\mathrm{C}$ [42]. The results given for the interactions Bonn A, B, and $C$ are very similar. This can be understood by the fact that the main difference between the three Bonn interactions is the strength of the $T=0$ tensor force [42], which has no influence on the neutron-neutron states with $T=1$. This result is also in consistent with the finding in pure neutron matter, where the equation of state calculated by RBHF with Bonn A, B, and C interactions are very close [53].
TABLE I. Total energy $E$ and rms radius $R_{N}$ of $N$-neutron drops in a $\mathrm{HO}$ trap $(\hbar \omega=10 \mathrm{MeV})$ calculated by RBHF theory using the interactions Bonn $\mathrm{A}, \mathrm{B}$, and $\mathrm{C}$.

\begin{tabular}{|c|c|c|c|c|c|c|}
\hline \multirow[b]{2}{*}{$N$} & \multicolumn{2}{|c|}{ Bonn A } & \multicolumn{2}{|c|}{ Bonn B } & \multicolumn{2}{|c|}{ Bonn C } \\
\hline & $E(\mathrm{MeV})$ & $R_{N}(\mathrm{fm})$ & $E(\mathrm{MeV})$ & $R_{N}(\mathrm{fm})$ & $E(\mathrm{MeV})$ & $R_{N}(\mathrm{fm})$ \\
\hline 4 & 62.6 & 2.51 & 62.6 & 2.51 & 62.7 & 2.51 \\
\hline 6 & 94.2 & 2.51 & 94.3 & 2.51 & 94.4 & 2.51 \\
\hline 8 & 130.0 & 2.61 & 130.2 & 2.61 & 130.3 & 2.61 \\
\hline 10 & 183.5 & 2.73 & 183.8 & 2.74 & 183.9 & 2.74 \\
\hline 12 & 231.2 & 2.80 & 231.6 & 2.81 & 231.8 & 2.81 \\
\hline 14 & 275.4 & 2.84 & 275.9 & 2.85 & 276.2 & 2.85 \\
\hline 16 & 320.2 & 2.89 & 321.0 & 2.90 & 321.4 & 2.90 \\
\hline 18 & 373.2 & 2.97 & 374.3 & 2.98 & 374.7 & 2.98 \\
\hline 20 & 418.1 & 3.02 & 419.3 & 3.03 & 419.7 & 3.03 \\
\hline 22 & 485.5 & 3.08 & 487.0 & 3.08 & 487.4 & 3.08 \\
\hline 24 & 546.9 & 3.12 & 548.7 & 3.13 & 549.2 & 3.13 \\
\hline 26 & 606.4 & 3.16 & 608.5 & 3.17 & 609.1 & 3.17 \\
\hline 28 & 663.9 & 3.19 & 666.3 & 3.20 & 666.9 & 3.20 \\
\hline
\end{tabular}

In Fig. 3, we show the total energy in units of $\hbar \omega N^{4 / 3}$ for $N$-neutron drops (with $N$ from 4 to 50) in a $\mathrm{HO} \operatorname{trap}(\hbar \omega=10$ $\mathrm{MeV}$ ) calculated by RBHF theory using the Bonn interactions. For the cases of open shells, the filling approximation is used. The results are compared with quantum Monte Carlo (QMC)

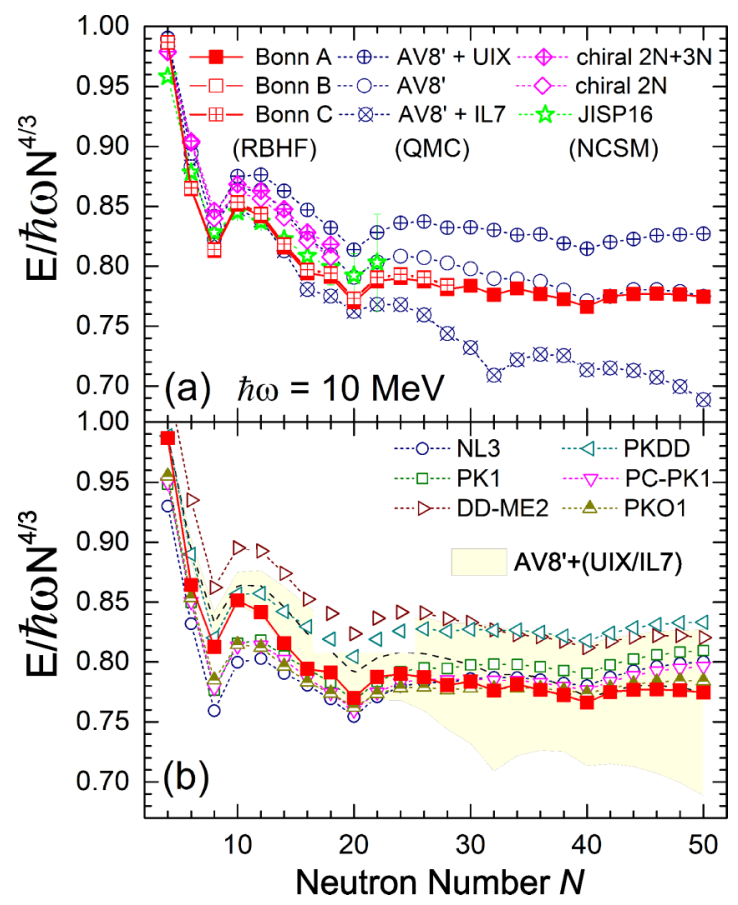

FIG. 3. Total energy in units of $\hbar \omega N^{4 / 3}$ for $N$-neutron drops in a HO trap $(\hbar \omega=10 \mathrm{MeV})$ calculated by RBHF theory using the interaction Bonn A (a) in comparison with QMC calculations [26,33] using the interactions AV8' + UIX, AV8', and AV8' + IL7, with NCSM calculations [26,27] using chiral $2 N+3 N$ forces, chiral $2 N$ forces, and the interaction JISP16; and (b) in comparison with results based on relativistic density functionals $[37,54]$. The shaded area indicates the QMC results. 
calculations [26,33] based on the interactions AV8' + UIX, AV8', and AV8' + IL7, with no-core shell-model (NCSM) calculations [26,27] based on chiral $2 N+3 N$ forces, on chiral $2 N$ force, and on JISP16, with calculations that use relativistic density functionals [37,54].

As has already been discussed above, the results of Bonn $\mathrm{A}, \mathrm{B}$, and $\mathrm{C}$ are very similar. Therefore, in later discussions we use Bonn A only. By comparing with QMC and NCSM calculations, the results of RBHF with the interaction Bonn A are similar to the results of the JISP16 interaction, and AV8' + IL7 (for $N \leqslant 14$ ), and getting closer to AV8' for $N \geqslant 20$. This similarity is favorable because JISP16 is a phenomenological nonlocal $N N$ interaction which can reproduce scattering data and gives a good description for light nuclei [55,56]. On the other hand, AV8' + IL7 gives a better description for light nuclei up to $A=12$ than AV8' or AV8' + UIX, but gives too much over-binding for pure neutron matter at higher densities [26,57].

In comparison with relativistic density functional calculations, we take four types of functionals, which cover a wide range of relativistic density functionals presently on the market:

(1) nonlinear meson couplings NL3 [58], PK1 [59];

(2) density-dependent meson couplings DD-ME2 [60], PKDD [59];

(3) point-coupling PC-PK1 [61];

(4) functional for RHF-calculations, PKO1 [54] (which includes tensor force).

Because there is no pairing in the RBHF calculation, we do not include pairing in the relativistic density functional calculations. We would like to mention that, generally, by including pairing effects, the binding energies of open-shell neutron drops would get larger, while for closed-shell drops they are unchanged. The overall energy as a function of neutron number $N$ will be smoother, as demonstrated in Ref. [37]. However, the effect is too small to be significant in the figure, so we do not plot it out and, more importantly, for the purpose of consistency with RBHF calculation, we use the results without pairing in the rest of our discussion.

From Fig. 3(b) it can be seen that the binding energies given by RBHF are generally bigger than those given by DD-ME2 and PKDD. For $N=8, \mathrm{RBHF}$ is close to PKDD, but getting closer to PK1 from $N=14$ to 26 , and closer to PC-PK1, NL3, and PKO1 from $N=28$ to 36. From $N=20$ on, the results of RBHF and DD-ME2 are close to a horizontal line, while the others have a small increasing tendency. The microscopic results obtained by RBHF can be a guidance for future density functionals. For example, the neutron-neutron interaction might be too repulsive in DD-ME2, whereas it might be too attractive in NL3 when the neutron number $N$ is small and then become too repulsive as $N$ becomes large.

Since in these calculations the $\hbar \omega=10 \mathrm{MeV}$ HO external field is chosen, they all show the HO magic numbers 8,20 , and 40. Beside these magic numbers, the results of RBHF indicate a subshell closure at $N=32$, similar to the results of AV8' + IL7. The subshell closure at $N=32$ is not significant for AV8' and does not exist for AV8' + UIX. For the $N=28$ subshell

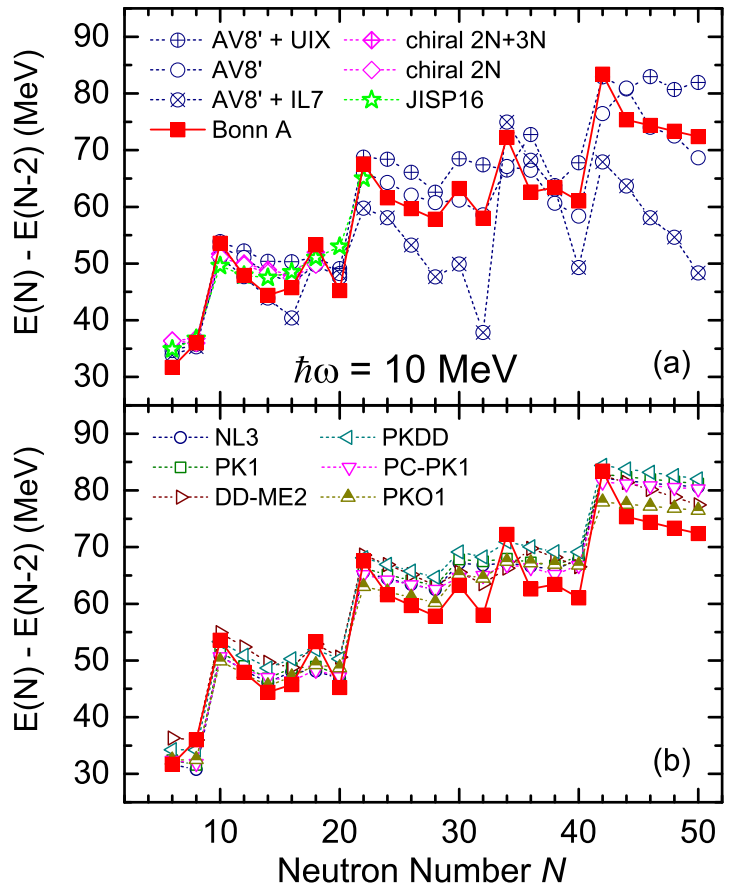

FIG. 4. Two-neutron energy difference of $N$-neutron drops in a $\mathrm{HO}$ trap $(\hbar \omega=10 \mathrm{MeV})$ calculated by RBHF theory using Bonn A interaction (a) in comparison with QMC calculations [26,33] using the interactions AV8' + UIX, AV8', and AV8' + IL7, with NCSM calculations using the interactions chiral $2 N+3 N$ force, chiral $2 N$ force [27], and JISP16 [26]; and (b) in comparison with relativistic density functionals [37].

closure, the results of Bonn A and AV8' + UIX show a small hint, while AV8' and AV8' + IL7 do not show it. On the other hand, all the relativistic density functionals only show the HO magic number $8,20,40$, and no clear subshells closures for $N=28$ or 32 .

To see the shell structure more clearly, we present in Fig. 4 the negative two-neutron separation energies $E(N)-E(N-$ 2 ) for the above calculations. The HO magic number 8, 20, 40 are clearly shown in all calculations. But the traditional subshell at $N=28$ in a finite system does not show up clearly in neutron drops. On the other hand, the results of AV8' + IL7 show a prominent subshell closure at $N=32$, while RBHF with Bonn A shows a modest but also clear closure at that neutron number.

By looking into Fig. 4(b), it can be seen that the results of relativistic density functionals are much smoother than those of the $a b$ initio calculations. In particular, it is interesting to see that these density functionals do not show clear subshell structure at $N=32$ and only a small closure at $N=28$. The subshell closure is related to the underlying single-particle spectra. Taking the $N=32$ subshell as an example, the $2 p_{3 / 2}$ state is just fully occupied and from $N=34$ on the $1 f_{5 / 2}$ state (for certain cases $2 p_{1 / 2}$ ) will begin to be occupied. Therefore, the gap between single-particle states $1 f_{5 / 2}$ (or $2 p_{1 / 2}$ ) and $2 p_{3 / 2}$ is a reflection of how strong the $N=32$ subshell is; see also the discussions on the single-particle energies in Sec. IVE. For RBHF with Bonn A, this gap is $3.047 \mathrm{MeV}$ in the $N=34$ 


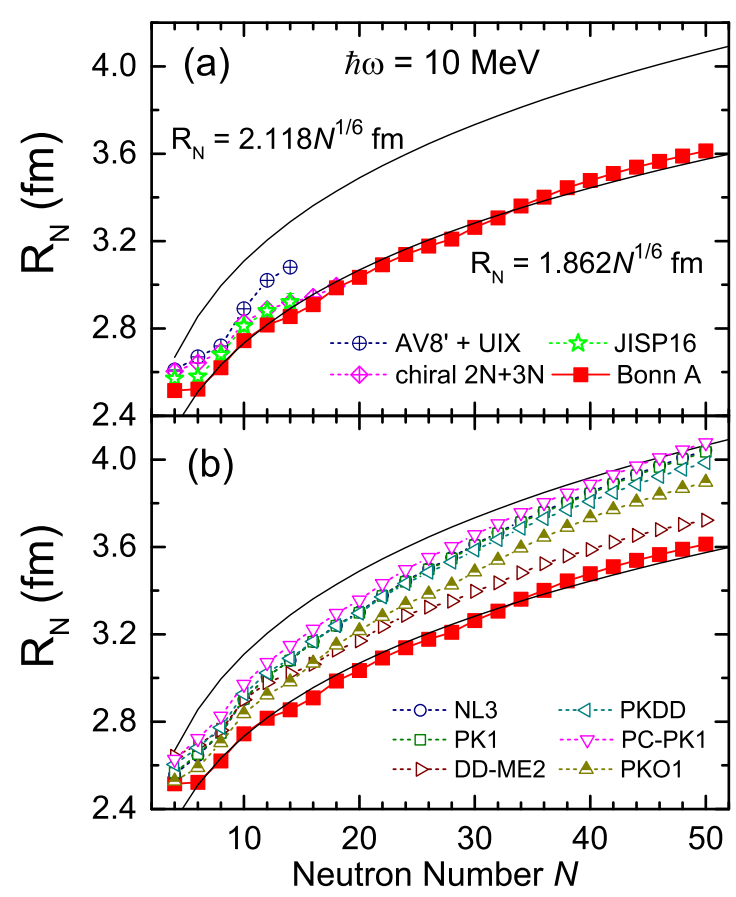

FIG. 5. Radii of $N$-neutron drops in a $\mathrm{HO}$ trap $(\hbar \omega=10 \mathrm{MeV})$ calculated by RBHF theory using the interaction Bonn A (a) in comparison with QMC calculations using the interaction AV8' + UIX [33], with NCSM calculations [26,27] using the chiral $2 N+3 N$ force and the interaction JISP16; and (b) in comparison with relativistic density functionals [37]. Further details are given in the text.

drop, while other relativistic density functionals give values ranging from $0.401 \mathrm{MeV}$ (NL3) to $2.127 \mathrm{MeV}$ (DD-ME2), which are much smaller than that of RBHF. Therefore the $N=32$ subshell closure given by RBHF is stronger than those of relativistic density functionals. This might be a hint that some parts of the effective Lagrangian are missing in these models. However, to understand the underlying detail, a decomposition of the $G$ matrix into different channels (scalar, vector, tensor, and so on) and a careful comparison with various density functionals are indispensable. Work along this direction is in progress.

\section{B. Radii}

Figure 5 shows the rms radii of $N$-neutron drops in a HO trap $(\hbar \omega=10 \mathrm{MeV}$ ) calculated in the framework of RBHF theory using the interaction Bonn A. In the upper panel the results are compared with QMC calculations based on the interactions AV8' + UIX [33], with NCSM calculations [26,27] based on the chiral $2 N+3 N$ force and based on the JISP16 force. In the lower panel these results are compared with calculations using relativistic density functionals. The black line in the upper and the lower panels $R_{N}=2.118 N^{1 / 6} \mathrm{fm}$ is obtained by solving for free fermions in a $\hbar \omega=10 \mathrm{MeV}$ HO trap using the ThomasFermi approximation, which can be derived as

$$
R_{N}=\left(\frac{3^{4 / 3}}{4} \frac{\hbar}{M \omega}\right)^{1 / 2} N^{1 / 6}
$$

TABLE II. Root mean square radius $R_{N}$ of $N=50$ neutron drop in a $\mathrm{HO}$ trap $(\hbar \omega=10 \mathrm{MeV})$ calculated by RBHF theory using the interaction Bonn A. The asymmetry energy $a_{\text {sym }}$ and slope parameter $L$ calculated in nuclear matter [63-65] are also listed. They are compared with results of relativistic density functionals NL3 [58], PK1 [59], DD-ME2 [60], PKDD [59], PC-PK1 [61], and PKO1 [54].

\begin{tabular}{lccc}
\hline \hline & $R_{N=50}(\mathrm{fm})$ & $a_{\text {sym }}(\mathrm{MeV})$ & $L(\mathrm{MeV})$ \\
\hline Bonn A & 3.61 & $34.8[63-65]$ & $71[65]$ \\
NL3 & 4.04 & 36.6 & 119 \\
PK1 & 4.04 & 37.6 & 116 \\
DD-ME2 & 3.72 & 32.3 & 51 \\
PKDD & 3.99 & 36.8 & 90 \\
PC-PK1 & 4.08 & 35.6 & 113 \\
PKO1 & 3.90 & 34.4 & 98 \\
\hline \hline
\end{tabular}

For $M=938.926 \mathrm{MeV}$ and $\hbar \omega=10 \mathrm{MeV}$, one finds a factor $2.118 \mathrm{fm}$ in front of $N^{1 / 6}$. The black line $R_{N}=1.862 N^{1 / 6} \mathrm{fm}$ is obtained by fitting to the results of Bonn A from $N=6$ to 50 .

Generally, all the calculated radii fulfill the relationship $N^{1 / 6}$ as a function of $N$. In all selected calculations, RBHF with Bonn A gives the smallest radii. By comparing with other calculations in Figs. 5(a) and 3(a), we find that while AV8' + UIX gives the smallest binding energies, it also gives the largest radii. Even though the energies given by JISP16 are similar to those of Bonn A, the radii given by JISP16 are larger than those of Bonn A. The radii of relativistic density functionals in Fig. 5(b) are much larger than those of RBHF, even though some of their binding energies are larger than RBHF before $N=20$ in Fig. 3(b).

It is known (Ref. [62]) that the relativistic density functionals without density-dependence in the isovector channel show too-large neutron radii in realistic nuclei and we observe this for the neutron drops, too. The relation between the slope parameter $L$ and the neutron skin is well known. For neutron drops this is also discussed in Ref. [37].

For a better comparison, we list the radius of the $N=50$ neutron drop calculated by RBHF theory using the interaction Bonn A in Table II. The asymmetry energy $a_{\text {sym }}$ and slope parameter $L$ calculated in nuclear matter [63-65] have also been listed. They are compared with results of the relativistic density functionals. It can be seen that, in general, the radius of a neutron drop is large if $a_{\text {sym }}$ or $L$ is large, although in detail small discrepancies exist. For example, DD-ME2 gives the smallest $a_{\text {sym }}$ and $L$, and its radius is indeed the smallest among those of relativistic density functionals, but still larger than that of Bonn A. The radius of PC-PK1 is the largest, and its $a_{\text {sym }}$ or $L$ is large, but not the largest, which is slightly smaller than those of NL3 and PK1.

In Ref. [37], a strong linear correlation has been found between the neutron skin thickness $\Delta r_{n p}$ and the rms radius $R_{N}$ of $N$-neutron drops in an external HO field. Figure 6 shows the linear correlation between the neutron skin thickness of ${ }^{48} \mathrm{Ca}$ and the radius of $N=20$ neutron drops in a $\hbar \omega=10 \mathrm{MeV}$ $\mathrm{HO}$ external field as given in Ref. [37]. The black circle and square symbols are calculated with different nonrelativistic and 


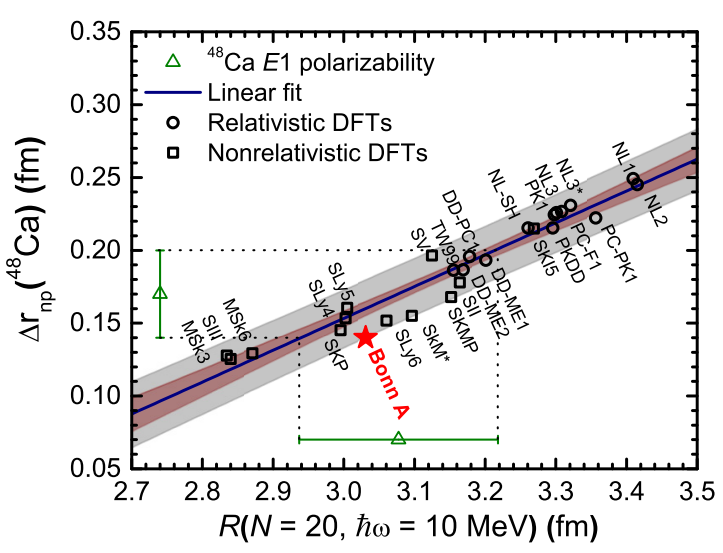

FIG. 6. Neutron skin thickness $\Delta r_{n p}$ of ${ }^{48} \mathrm{Ca}$ and the rms radius $R$ of $N=20$ neutron drop in a $\mathrm{HO} \operatorname{trap}(\hbar \omega=10 \mathrm{MeV})$ calculated by RBHF theory using the interaction Bonn A (red star), in comparison with results obtained by various density functionals [37]. The datum of $\Delta r_{n p}$ is obtained by measuring the electric-dipole polarizability of ${ }^{48} \mathrm{Ca}$ [66]. The blue line is the linear fit to the results of density functionals, and the inner (outer) colored regions depict the $95 \%$ confidence (prediction) intervals of the linear regression [37].

relativistic density functionals, and the blue line is obtained by fitting to these results [37]. The inner (outer) colored regions depict the $95 \%$ confidence (prediction) intervals of the linear regression.

The red star in Fig. 6 is calculated by RBHF theory using the interaction Bonn A. The datum of the neutron skin thickness of ${ }^{48} \mathrm{Ca}$ is obtained by measuring the electric-dipole polarizability in Ref. [66]. It can be seen that the neutron skin thickness of ${ }^{48} \mathrm{Ca}$ given by RBHF $\Delta r_{n p}=0.14 \mathrm{fm}$ is located within the error bar of experimental data, which is also consistent with the $0.12 \leqslant \Delta r_{n p} \leqslant 0.15$ fm given by coupled-cluster calculations using the interaction $\mathrm{NNLO}_{\text {sat }}$ [67].

Apart from the linear correlation between $\Delta r_{n p}$ of ${ }^{48} \mathrm{Ca}$ and radius of $N=20$ neutron drops in Fig. 6, similar correlations can be found in other cases; for example, for $\Delta r_{n p}$ of ${ }^{208} \mathrm{~Pb}$ or other numbers of $N$. Using these linear correlations, the experimental data of neutron skins of ${ }^{48} \mathrm{Ca}$ and ${ }^{208} \mathrm{~Pb}$ can be mapped to the data of radii of neutron drops with different numbers of $N$ [37], and the results are shown by the green symbols in Fig. 7. In this way, the study of the neutron skin of heavy nuclei can be linked to the study of the radius of neutron drops, while the latter is much easier to be accessed by different ab initio calculations.

In Fig. 7, we show the radii for $N=20,14$, and 8 neutron drops calculated by RBHF theory using the interaction Bonn A (red dashed lines), in comparison with data (green symbols) determined from the linear correlations with the neutron skin thicknesses of ${ }^{208} \mathrm{~Pb}$ and ${ }^{48} \mathrm{Ca}$ [37], and other $a b$ initio calculations (blue dotted lines) $[26,33,34]$. For $\Delta r_{n p}$ of ${ }^{208} \mathrm{~Pb}$, the data come from different measurements with antiprotonic atoms [68] (circles), pion photoproduction [69] (squares), and electric-dipole polarizability [70] (diamonds); for $\Delta r_{n p}$ of ${ }^{48} \mathrm{Ca}$, the datum comes from the measurement of the electric dipole polarizability [66] (triangles). For the local chiral forces $\mathrm{N}^{2} \mathrm{LO}$ from Refs. [71,72], we present the results

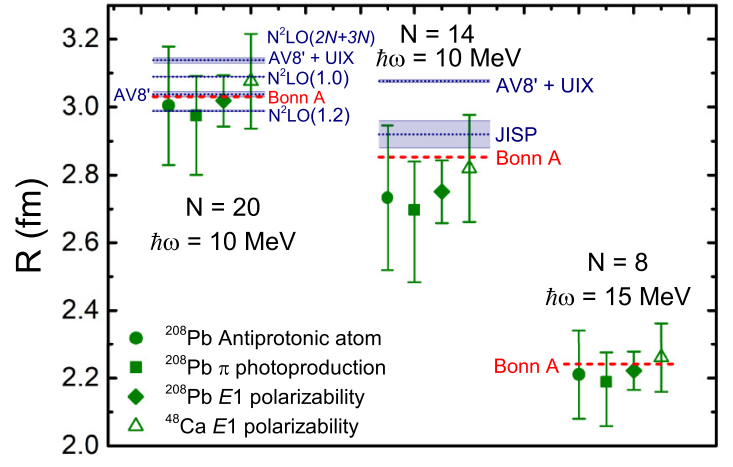

FIG. 7. Radii for $N=20,14$, and 8 neutron drops calculated by RBHF theory using the interaction Bonn A (red dashed line), in comparison with data (green symbols) determined from the linear correlations with the neutron skin thicknesses of ${ }^{208} \mathrm{~Pb}$ and ${ }^{48} \mathrm{Ca}$ [37], and other ab initio calculations (blue dots) [26,33,34]. The blue-colored regions denote theoretical uncertainties.

of a two-body force with a cutoff $R_{0}=1.0$ and $1.2 \mathrm{fm}$, and a two-body plus three-body force $(2 N+3 N)$ with a cutoff $R_{0}=1.2 \mathrm{fm}$ [34]. Theoretical uncertainties are denoted by the blue-colored regions. There is no particular reason to choose $N=20,14$, and 8 neutron drops, as long as the central density of the neutron drop does not differ too much from the saturation density $\left(\approx 0.16 \mathrm{fm}^{-3}\right)$ [37].

It can be seen that the radii obtained in RBHF calculations with the interaction Bonn $\mathrm{A}$ are in good agreement with the data determined from the linear correlations with the neutron skin thicknesses. In comparison with other $a b$ initio calculations, AV8' + UIX shows more repulsion and gives larger radii, as expected from the energies shown in Fig. 3. For the $2 N$ local chiral forces $\mathrm{N}^{2} \mathrm{LO}$, the softer interaction with a cutoff radius $R_{0}=1.2 \mathrm{fm}$ gives a smaller radius and the harder one with $R_{0}=1.0 \mathrm{fm}$ gives a larger radius. When including the $3 \mathrm{~N}$ force for $\mathrm{N}^{2} \mathrm{LO}$, the radius gets larger by $0.05 \mathrm{fm}$ and is in the same position as AV8' + UIX in Fig. 7.

\section{Density distribution}

Figure 8 shows the density distributions of $N$-neutron drops in a HO trap $(\hbar \omega=10 \mathrm{MeV})$ calculated by RBHF theory using the interaction Bonn A. With given HO strength, the neutron density gets saturated around $0.14-0.17 \mathrm{fm}^{-3}$. For neutron drops with $N=40$ or $N=50$ there is a bubble structure in the center. This can be understood from the occupation of single-particle states. Near $N=20$, the $2 s_{1 / 2}$ state has just been occupied and it has a large contribution to the central density. From $N=20$ to $N=50$, the $1 f, 2 p$, and $1 g_{9 / 2}$ states start to be occupied and, because their angular momentum $l \neq 0$, the density begins to shift outward. Similar to the proton bubble structure in ${ }^{34} \mathrm{Si}$, where the proton $2 s_{1 / 2}$ state is empty, and just to be occupied in the next nucleus ${ }^{36} \mathrm{~S}$ [73].

\section{Single-particle potential}

The single-particle potential in the RHF equation (10) is a nonlocal potential. However, for a given single-particle wave function, one can construct an equivalent local potential for 


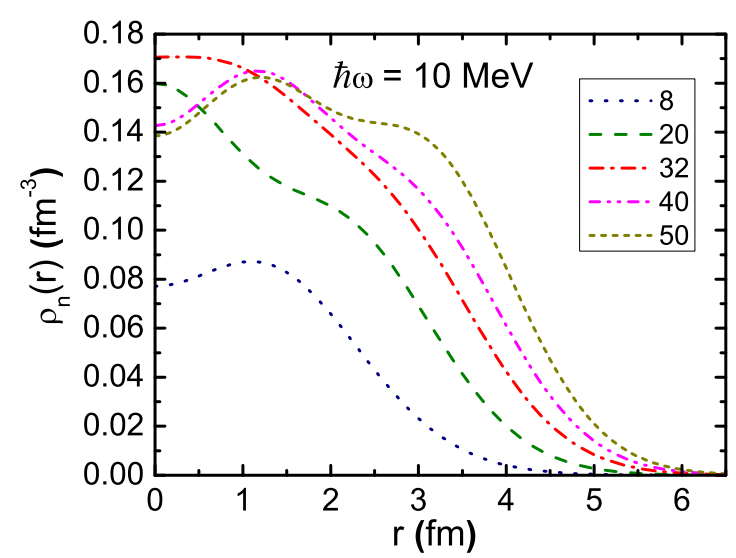

FIG. 8. Density distributions of $N$-neutron drops in a HO trap $(\hbar \omega=10 \mathrm{MeV}$ ) calculated by RBHF theory using the interaction Bonn A.

this state by using the Dirac equation. For spherical symmetry, one has the radial equation

$$
\left(\begin{array}{cc}
M+\Sigma(r) & -\frac{d}{d r}+\frac{\kappa}{r} \\
\frac{d}{d r}+\frac{\kappa}{r} & -M+\Delta(r)
\end{array}\right)\left(\begin{array}{c}
F_{a}(r) \\
G_{a}(r)
\end{array}\right)=e_{a}\left(\begin{array}{c}
F_{a}(r) \\
G_{a}(r)
\end{array}\right)
$$

where $\Sigma=V+S$ and $\Delta=V-S$ are the sum and the difference of vector and scalar potentials, the quantum number $\kappa$ is defined as $\kappa= \pm(j+1 / 2)$ for $j=l \mp 1 / 2$. Then one finds

$$
\begin{aligned}
& \Sigma_{a}(r)=e_{a}-M+\left(\frac{d G_{a}(r)}{d r}-\frac{\kappa}{r} G_{a}(r)\right) F_{a}^{-1}(r), \\
& \Delta_{a}(r)=e_{a}+M-\left(\frac{d F_{a}(r)}{d r}+\frac{\kappa}{r} F_{a}(r)\right) G_{a}^{-1}(r) .
\end{aligned}
$$

Figure 9 shows this localized single-particle potential $\Sigma_{1 s 1 / 2}(r)$ for the $1 s_{1 / 2}$ state of $N$-neutron drops in a HO trap $(\hbar \omega=10 \mathrm{MeV})$ calculated by RBHF theory using the interaction Bonn A. As $r$ increases, the single-particle potentials approach the external HO potential. The central potential is negative and decreases as $N$ increases. This is a consequence

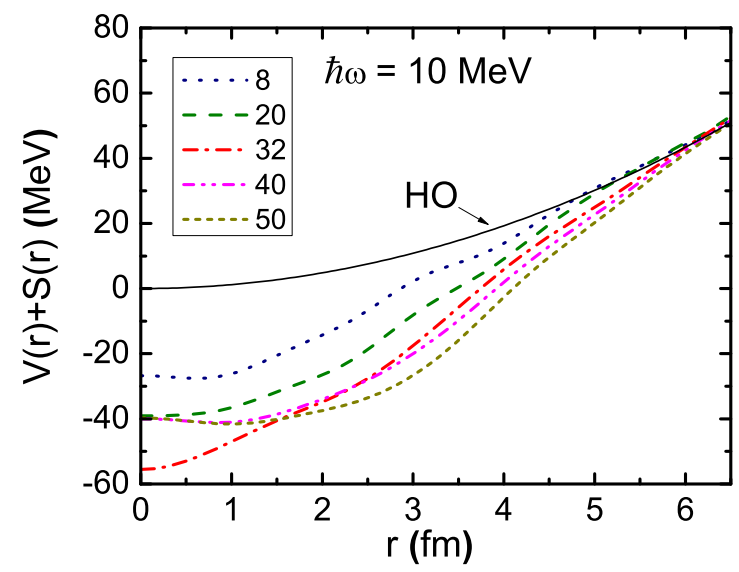

FIG. 9. Localized $1 s_{1 / 2}$ single-particle potential of $N$-neutron drops in a $\mathrm{HO}$ trap $(\hbar \omega=10 \mathrm{MeV})$ calculated by RBHF theory using the interaction Bonn A. The black line is the external HO potential.

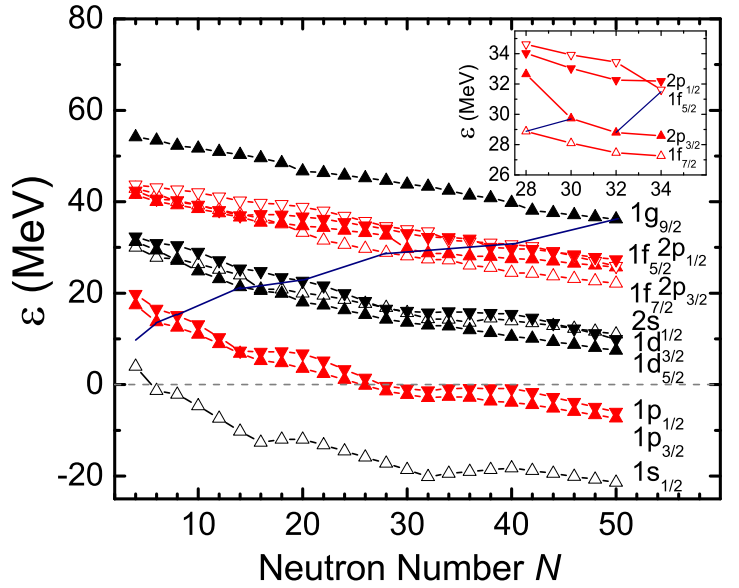

FIG. 10. Single-particle energies of $N$-neutron drops in a HO trap ( $\hbar \omega=10 \mathrm{MeV}$ ) as a function of $N$ calculated by RBHF theory using the interaction Bonn A. The blue line represents the Fermi surface.

of the attractive interaction between the neutrons. Similar to the density distribution shown in Fig. 8, the single-particle potential saturates as $N$ increases to 20 and the potential depth with respect to the potential of the $\mathrm{HO}$ trap is about $-40 \mathrm{MeV}$.

\section{E. Single-particle energies}

In Fig. 10, we show the single-particle energies of $\mathrm{N}$ neutron drops in a $\mathrm{HO}$ trap $(\hbar \omega=10 \mathrm{MeV})$ as a function of $N$ calculated by RBHF theory using the interaction Bonn A. The blue line represents the Fermi surface. The filling approximation is used for open-shell neutron drops.

Generally, the single-particle energies decrease as the number of neutron increases, because the potential becomes wider with increasing neutron number $N$. The inset in Fig. 10 shows the details of $1 f$ and $2 p$ orbits in the region between $N=28$ and $N=34$. We observe how the traditional subshell closure at $N=28$ disappears and a new closure at $N=32$ appears in neutron drops.

Another interesting phenomenon can be seen in the upper panel of Fig. 11. It shows the evolution of spin-orbit (SO)

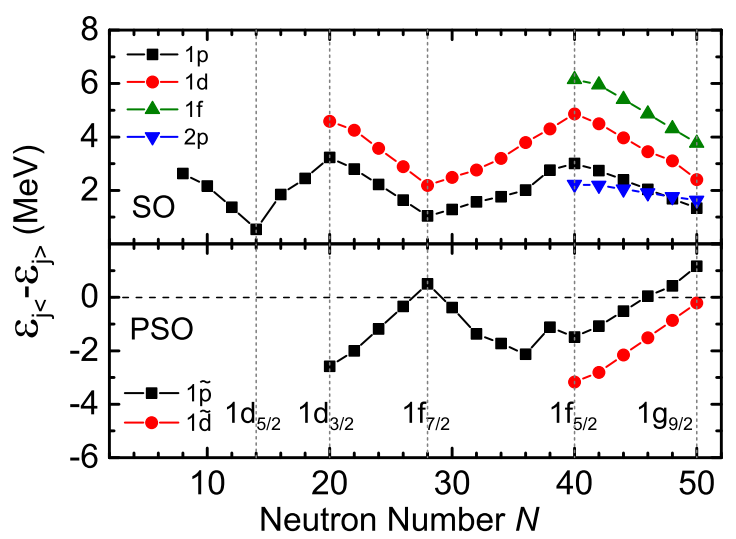

FIG. 11. Neutron spin-orbit and pseudospin-orbit splittings of $N$-neutron drops in a $\mathrm{HO} \operatorname{trap}(\hbar \omega=10 \mathrm{MeV})$ as a function of $N$ calculated by RBHF theory using the interaction Bonn A. 


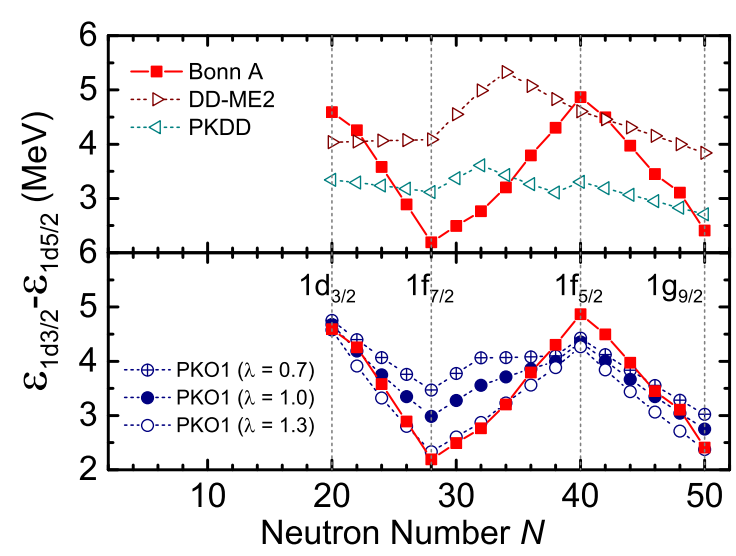

FIG. 12. Neutron spin-orbit for the $1 d$ orbit as a function of the neutron number $N$. Full RBHF calculations with the interaction Bonn A are compared with the two conventional density functionals PKDD and DD-ME2 without tensor contributions (upper panel) and with the RHF-functional PKO1, which contains a tensor contribution in the Fock term of the pion-exchange force (lower panel). Its strength $f_{\pi}$ is slightly varied (by a factor $\lambda$ ) as compared with the strength in PKO1.

splitting as the neutron number increases. The SO splitting decreases as the next-higher $j=j_{>}=l+1 / 2$ orbit is filled and reaches a minimum when this orbit is fully occupied. As the number of neutron continues to increase, the $j=j_{<}=l-1 / 2$ orbit begins to be occupied and the SO splitting increases.

A similar effect has been found by Otsuka et al. [74]. They explained it in terms of the monopole effect of the tensor force, which produces an attraction between a proton in a SO aligned orbit with $j=j_{>}=l+1 / 2$ and a neutron in a SO anti-aligned orbit with $j^{\prime}=j_{<}^{\prime}=l^{\prime}-1 / 2$ and a repulsion between the same proton and a neutron in a $\mathrm{SO}$ aligned orbit with $j^{\prime}=j_{>}^{\prime}=l^{\prime}+1 / 2$.

As discussed in the same paper [74], a similar mechanism with smaller amplitude exists also for the tensor interaction between neutrons with $T=1$. The behavior of the SO splitting in Fig. 11 has been explained qualitatively in a similar way in Ref. [28]. Consider, for instance, the decreasing of the $1 d \mathrm{SO}$ splitting from $N=20$ to $N=28$. Above $N=20$ the neutrons fill into the SO aligned orbit $1 f_{7 / 2}$. They show repulsion with the SO aligned $1 d_{5 / 2}$ neutrons and attraction with the SO antialigned $1 d_{3 / 2}$ neutrons. This means that, by filling neutrons into the $1 f_{7 / 2}$ shell, the $1 d_{5 / 2}$ orbit is shifted upward and the $1 d_{3 / 2}$ is shifted downward, reducing the $1 d$ SO splitting more and more. Above $N=28$ the neutrons fill into the $\mathrm{SO}$ anti-aligned states $2 p_{1 / 2}$ and $1 f_{5 / 2}$. They interact with the $1 d$ neutrons in the opposite way and increase the $1 d \mathrm{SO}$ splitting.

To study whether the characteristic variation of the SO splitting shown in the upper panel of Fig. 11 is indeed connected with the properties of the tensor force, the results of RBHF with Bonn A interaction have been compared with various relativistic density functionals, with and without tensor force [28]. It has been found that the tensor force is the major reason for this pattern of the evolution of SO splittings. In Fig. 12 our RBHF results for the $1 d$ spin-obit splitting with results obtained with phenomenological density functionals from the literature. Most of them, such as, for instance, the functionals
DD-ME2 [60] or PKDD [59], are based on relativistic Hartree calculations and do not include a tensor term and, indeed, as shown in the upper panel of Fig. 12, these functionals are not able to reproduce the specific pattern for the $1 d$ splitting.

On the other hand, the Hartree-Fock functionals PKO1 [54] and PKA1 [75] include in the Fock term of the pion- and of the $\rho$-meson exchange-force tensor terms, PKO1 only for the pion, and PKA1 for both the pion and for the $\rho$. In the lower panel of Fig. 12 it is clearly seen that the SO splitting produced by the density functional PKO1 closely follows the pattern of our $a b$ initio RBHF calculations. By changing the strength of the pion-exchange, i.e., by changing the size of the corresponding tensor term, it is clearly seen that the size of the tensor effect significantly depends on the value of $\lambda$, where the cases of $\lambda=0.7,1.0$, and 1.3 are shown in the figure. For $\lambda=1$ we have the results of the density functional PKO1. With $\lambda=1.3$, the specific evolution pattern of the SO splitting generated by the $a b$ initio RBHF calculations can be nicely reproduced. As in the case of the shell-model calculations of the Otsuka et al. [74], it is the tensor which causes the peculiar behavior of the SO splitting of the drops with increasing neutron number. The pattern for the functional PKA1 is similar, therefore we did not present it here.

The pseudospin-orbit (PSO) splitting [76-81] in the lower panel of Fig. 11 shows a similar pattern, but in the direction opposite that of the SO splitting. This can also be understood by the effect of the tensor force. Taking the PSO splitting of $1 \tilde{p}$ as an example, it is defined as $E_{\tilde{j}<}-E_{\tilde{j}>}=E_{2 s 1 / 2}-E_{1 d 3 / 2}=$

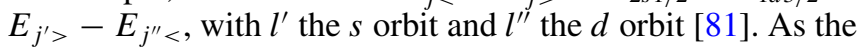
$j_{>}$orbit, for example $1 f_{7 / 2}$, is being occupied, there will be a repulsion for $j_{>}^{\prime}=2 s_{1 / 2}$ and an attraction for $j^{\prime \prime}{ }_{<}=1 d_{3 / 2}$. Thus, the PSO splitting of $1 \tilde{p}$ will increase and even become positive at $N=28$, when $j_{>}=1 f_{7 / 2}$ is fully occupied. The situation becomes more complicated when the $2 p$ orbit is being filled and the monopole effect of the tensor force is less prominent.

\section{SUMMARY}

We have studied neutron drops confined in an external field of oscillator shape using relativistic Brueckner-Hartree-Fock theory with the relativistic $N N$ interactions Bonn A, B, and $C$ [42]. The results are compared with other nonrelativistic $a b$ initio and with relativistic density functional calculations.

First, we checked the convergence of RBHF calculations for neutron drops with $N=8,20,28$, and 50 with respect to the single-particle energy cutoff $\varepsilon_{\text {cut }}$ and found good convergence at $900 \mathrm{MeV}$. This energy cutoff is smaller than the cutoff in finite nuclei at $1100 \mathrm{MeV}$. This can be understood by the lack of the $T=0$ tensor force in neutron drops. We also showed that this energy cutoff does not depend on the number of particles.

We calculated $N=4$ to 28 neutron drops with the interactions Bonn $\mathrm{A}, \mathrm{B}$, and $\mathrm{C}$ and found similar results for these three interactions. We compared for Bonn A our RBHF results for $N=4$ to $N=50$ neutrons with other ab initio calculations and with various relativistic density functionals. The harmonic-oscillator magic numbers 8,20 , and 40 show up in all the selected results, but the subshell closures at $N=28$ and $N=32$ strongly depend on the interactions. There is a 
slight sign of a subshell closure at $N=28$ for AV8' + UIX, but no sign for the other interactions. For $N=32$, AV8' + IL7 shows a clear subshell closure, while the subshell closure for RBHF with Bonn A and for AV8' is smaller but still significant. On the other hand, relativistic density functionals show only the HO magic numbers.

We also studied the radii of neutron drops in a HO trap. With increasing $N$ they follow closely the $N^{1 / 6}$ rule, which can be derived for noninteracting neutrons in the Thomas-Fermi approximation. While the energies of RBHF with Bonn A are similar to those of the JISP16 interaction, the radii of RBHF are smaller. On the other hand, the radii calculated by various relativistic density functionals are all larger than $\mathrm{RBHF}$ with Bonn A, even though the energies found in RBHF are among these obtained with the density functionals. However, the smaller radii given by $\mathrm{RBHF}$ are in good agreement with pseudodata derived from the experimental neutron skin thickness of ${ }^{48} \mathrm{Ca}$ and ${ }^{208} \mathrm{~Pb}$. These pseudodata are derived from the strong linear correlation found in Ref. [37] between the radius of a fixed neutron drop and the neutron skin thickness of a specific nucleus for various nuclear density functionals. In particular we have calculated the neutron skin thickness of ${ }^{48} \mathrm{Ca}$ by $\mathrm{RBHF}$ with Bonn $\mathrm{A}$ and the value is consistent with recent experimental datum [66] and coupled-cluster calculations [67].

We show the density distribution of neutron drops with $N=8,20,32,40$, and 50 and find that the density gets saturated around $0.14-0.17 \mathrm{fm}^{-3}$. Similarly, we calculated the local equivalent single-particle potentials for the $1 s_{1 / 2}$ states and find also saturation for $N \geqslant 20$ at a potential depth of around $-40 \mathrm{MeV}$. These results depend on the strength of the external HO field. We used $\hbar \omega=10 \mathrm{MeV}$, and changing the strength will change the saturation properties.

Finally, we studied the evolution of the single-particle energies as a function of $N$. The disappearance of a subshell closures at $N=28$ and appearance at $N=32$ can be seen clearly. We also find that the evolution of the spin-orbit and the pseudospin-orbit splittings show a interesting pattern, which can be explained in a similar way by the tensor force as has been done in nuclei in Ref. [74].

The results of RBHF show many interesting features and can provide important information for future density functionals, especially in the area of neutron-rich exotic nuclei. We name below a few future guidelines:
(1) It is evident from the results on the spin-orbit splitting that we have to introduce a tensor term. We need a further study on several tensor terms (zero range, pion like, rho like) to find out which is the most appropriate.

(2) One could adjust the parameters of future relativistic density functionals not only to the conventional data on nuclear matter and finite nuclei, but also to the matrix elements of the $G$ matrix in specific nuclei.

(3) In a more systematic way, one could decompose the $G$ matrix into the different relativistic channels and to study which of them are important for specific types of nuclei.

(4) Applying external fields of various types and studying their influence on the RBHF-results will allow us to model the corresponding relativistic density functionals. An example would be the application of an external magnetic field in order to study the time-odd parts of the functionals. Another example would be the solution of half-infinite nuclear matter in the RBHF framework for the study of the surface properties of the functionals.

Of course, these are only examples and details have to be investigated in the future. However, it is evident that the knowledge of the $G$ matrix in finite systems opens a completely new field of investigation to improve the functionals.

So far, there is only one relativistic nucleon-nucleon force: the Bonn potential. With the recent progress in covariant chiral interactions $[82,83]$ it will be also interesting to study the neutron drops using RBHF theory with covariant chiral interactions.

\section{ACKNOWLEDGMENTS}

We thank Pengwei Zhao for discussions and providing his results. This work was partly supported by the National Key R\&D Program of China (Contract No. 2018YFA0404400), Natural Science Foundation of China under Grants No. 11335002, No. 11375015, and No. 11621131001, the Overseas Distinguished Professor Project from Ministry of Education of China No. MS2010BJDX001, the Research Fund for the Doctoral Program of Higher Education in China under Grant No. 20110001110087, and the DFG (Germany) cluster of excellence "Origin and Structure of the Universe" (www.universecluster.de). H.L. would like to thank the RIKEN iTHES project and iTHEMS program.
[1] M. Goeppert-Mayer, Phys. Rev. 75, 1969 (1949).

[2] O. Haxel, J. H. D. Jensen, and H. E. Suess, Phys. Rev. 75, 1766 (1949).

[3] B. Bastin, S. Grévy, D. Sohler, O. Sorlin, Z. Dombrádi, N. L. Achouri, J. C. Angélique, F. Azaiez, D. Baiborodin, R. Borcea et al., Phys. Rev. Lett. 99, 022503 (2007).

[4] O. Sorlin and M.-G. Porquet, Prog. Part. Nucl. Phys. 61, 602 (2008).

[5] D. Steppenbeck, S. Takeuchi, N. Aoi, P. D. M. Matsushita, H. Wang, H. Baba, N. Fukuda, S. Go, M. Honma, J. Lee et al., Nature (London) 502, 207 (2013).
[6] I. Tanihata, H. Hamagaki, O. Hashimoto, Y. Shida, N. Yoshikawa, K. Sugimoto, O. Yamakawa, T. Kobayashi, and N. Takahashi, Phys. Rev. Lett. 55, 2676 (1985).

[7] J. Meng and P. Ring, Phys. Rev. Lett. 77, 3963 (1996).

[8] J. Meng, Nucl. Phys. A 635, 3 (1998).

[9] D. Vautherin and D. M. Brink, Phys. Rev. C 5, 626 (1972).

[10] J. D. Walecka, Ann. Phys. (NY) 83, 491 (1974).

[11] J. Dechargé and D. Gogny, Phys. Rev. C 21, 1568 (1980).

[12] M. Bender, P.-H. Heenen, and P.-G. Reinhard, Rev. Mod. Phys. 75, 121 (2003). 
[13] S. Bogner, A. Bulgac, J. Carlson, J. Engel, G. Fann, R. Furnstahl, S. Gandolfi, G. Hagen, M. Horoi, C. Johnson et al., Comput. Phys. Commun. 184, 2235 (2013).

[14] B. D. Day, Rev. Mod. Phys. 39, 719 (1967).

[15] W. H. Dickhoff and C. Barbieri, Prog. Part. Nucl. Phys. 52, 377 (2004).

[16] D. Lee, Prog. Part. Nucl. Phys. 63, 117 (2009).

[17] L. Liu, T. Otsuka, N. Shimizu, Y. Utsuno, and R. Roth, Phys. Rev. C 86, 014302 (2012).

[18] B. R. Barrett, P. Navratil, and J. P. Vary, Prog. Part. Nucl. Phys. 69, 131 (2013).

[19] G. Hagen, T. Papenbrock, M. Hjorth-Jensen, and D. J. Dean, Rep. Prog. Phys. 77, 096302 (2014).

[20] J. Carlson, S. Gandolfi, F. Pederiva, S. C. Pieper, R. Schiavilla, K. E. Schmidt, and R. B. Wiringa, Rev. Mod. Phys. 87, 1067 (2015).

[21] H. Hergert, S. Bogner, T. Morris, A. Schwenk, and K. Tsukiyama, Phys. Rep. 621, 165 (2016).

[22] S. Shen, J. Hu, H. Liang, J. Meng, P. Ring, and S. Zhang, Chin. Phys. Lett. 33, 102103 (2016).

[23] S. Shen, H. Liang, J. Meng, P. Ring, and S. Zhang, Phys. Rev. C 96, 014316 (2017).

[24] B. S. Pudliner, A. Smerzi, J. Carlson, V. R. Pandharipande, S. C. Pieper, and D. G. Ravenhall, Phys. Rev. Lett. 76, 2416 (1996).

[25] S. K. Bogner, R. J. Furnstahl, H. Hergert, M. Kortelainen, P. Maris, M. Stoitsov, and J. P. Vary, Phys. Rev. C 84, 044306 (2011).

[26] P. Maris, J. P. Vary, S. Gandolfi, J. Carlson, and S. C. Pieper, Phys. Rev. C 87, 054318 (2013).

[27] H. D. Potter, S. Fischer, P. Maris, J. P. Vary, S. Binder, A. Calci, J. Langhammer, and R. Roth, Phys. Lett. B 739, 445 (2014).

[28] S. Shen, H. Liang, J. Meng, P. Ring, and S. Zhang, Phys. Lett. B 778, 344 (2018).

[29] R. B. Wiringa, V. G. J. Stoks, and R. Schiavilla, Phys. Rev. C 51, 38 (1995).

[30] B. S. Pudliner, V. R. Pandharipande, J. Carlson, and R. B. Wiringa, Phys. Rev. Lett. 74, 4396 (1995).

[31] A. Smerzi, D. G. Ravenhall, and V. R. Pandharipande, Phys. Rev. C 56, 2549 (1997).

[32] F. Pederiva, A. Sarsa, K. Schmidt, and S. Fantoni, Nucl. Phys. A 742, 255 (2004).

[33] S. Gandolfi, J. Carlson, and S. C. Pieper, Phys. Rev. Lett. 106, 012501 (2011).

[34] I. Tews, S. Gandolfi, A. Gezerlis, and A. Schwenk, Phys. Rev. C 93, 024305 (2016).

[35] D. R. Entem and R. Machleidt, Phys. Rev. C 68, 041001 (2003).

[36] E. Epelbaum, A. Nogga, W. Glöckle, H. Kamada, Ulf-G. Meißner, and H. Witała, Phys. Rev. C 66, 064001 (2002).

[37] P. W. Zhao and S. Gandolfi, Phys. Rev. C 94, 041302 (2016).

[38] M. R. Anastasio, L. S. Celenza, W. S. Pong, and C. M. Shakin, Phys. Rep. 100, 327 (1983).

[39] R. Brockmann and R. Machleidt, Phys. Lett. B 149, 283 (1984).

[40] B. ter Haar and R. Malfliet, Phys. Rep. 149, 207 (1987).

[41] W. Zuo, A. Lejeune, U. Lombardo, and J. Mathiot, Nucl. Phys. A 706, 418 (2002).

[42] R. Machleidt, in Advances in Nuclear Physics, edited by J. W. Negele and E. Vogt (Springer, New York, 1989), Vol. 19, pp. 189-376.

[43] S.-G. Zhou, J. Meng, and P. Ring, Phys. Rev. C 68, 034323 (2003).
[44] J. Meng, H. Toki, S. Zhou, S. Zhang, W. Long, and L. Geng, Prog. Part. Nucl. Phys. 57, 470 (2006).

[45] M. Serra, Ph.D. thesis, Technical University of Munich, 2001 (unpublished).

[46] K. A. Brueckner, C. A. Levinson, and H. M. Mahmoud, Phys. Rev. 95, 217 (1954).

[47] K. A. Brueckner, Phys. Rev. 96, 508 (1954).

[48] H. A. Bethe and J. Goldstone, Proc. R. Soc. London, Ser. A 238, 551 (1957).

[49] M. Baranger, in Nuclear Structure and Nuclear Reaction, Proceedings of the International School of Physics "Enrico Fermi," Course XL, Varenna, 1967, edited by M. Jean (Academic Press Inc., New York, 1969), Vol. 40, pp. 511-614.

[50] K. T. R. Davies, M. Baranger, R. M. Tarbutton, and T. T. S. Kuo, Phys. Rev. 177, 1519 (1969).

[51] R. Rajaraman and H. A. Bethe, Rev. Mod. Phys. 39, 745 (1967).

[52] P. Ring and P. Schuck, The Nuclear Many-Body Problem (Springer-Verlag, Berlin, 1980).

[53] G. Q. Li, R. Machleidt, and R. Brockmann, Phys. Rev. C 45, 2782 (1992).

[54] W.-H. Long, N. Van Giai, and J. Meng, Phys. Lett. B 640, 150 (2006).

[55] A. M. Shirokov, J. P. Vary, A. I. Mazur, and T. A. Weber, Phys. Lett. B 644, 33 (2007).

[56] P. Maris, J. P. Vary, and A. M. Shirokov, Phys. Rev. C 79, 014308 (2009).

[57] A. Sarsa, S. Fantoni, K. E. Schmidt, and F. Pederiva, Phys. Rev. C 68, 024308 (2003).

[58] G. A. Lalazissis, J. König, and P. Ring, Phys. Rev. C 55, 540 (1997).

[59] W. Long, J. Meng, N. Van Giai, and S.-G. Zhou, Phys. Rev. C 69, 034319 (2004).

[60] G. A. Lalazissis, T. Nikšić, D. Vretenar, and P. Ring, Phys. Rev. C 71, 024312 (2005).

[61] P. W. Zhao, Z. P. Li, J. M. Yao, and J. Meng, Phys. Rev. C 82, 054319 (2010).

[62] T. Nikšić, D. Vretenar, P. Finelli, and P. Ring, Phys. Rev. C 66, 024306 (2002).

[63] D. Alonso, and F. Sammarruca, Phys. Rev. C 67, 054301 (2003).

[64] E. N. E. van Dalen, C. Fuchs, and A. Faessler, Nucl. Phys. A 744, 227 (2004).

[65] T. Katayama and K. Saito, Phys. Rev. C 88, 035805 (2013).

[66] J. Birkhan, M. Miorelli, S. Bacca, S. Bassauer, C. A. Bertulani, G. Hagen, H. Matsubara, P. von Neumann-Cosel, T. Papenbrock, N. Pietralla et al., Phys. Rev. Lett. 118, 252501 (2017).

[67] G. Hagen, A. Ekström, C. Forsssn, G. R. Jansen, W. Nazarewicz, T. Papenbrock, K. A. Wendt, S. Bacca, N. Barnea, B. Carlsson et al., Nat. Phys. 12, 186 (2015).

[68] B. Klos, A. Trzcińska, J. Jastrzebski, T. Czosnyka, M. Kisieliński, P. Lubiński, P. Napiorkowski, L. Pieńkowski, F. J. Hartmann, B. Ketzer et al., Phys. Rev. C 76, 014311 (2007).

[69] Crystal Ball at MAMI and A2 Collaboration, C. M. Tarbert, D. P. Watts, D. I. Glazier, P. Aguar, J. Ahrens, J. R. M. Annand, H. J. Arends, R. Beck, V. Bekrenev, B. Boillat et al., Phys. Rev. Lett. 112, 242502 (2014).

[70] X. Roca-Maza, M. Brenna, G. Colo, M. Centelles, X. Vinas, B. K. Agrawal, N. Paar, and D. Vretenar, J. Piekarewicz, Phys. Rev. C 88, 024316 (2013).

[71] A. Gezerlis, I. Tews, E. Epelbaum, S. Gandolfi, K. Hebeler, A. Nogga, and A. Schwenk, Phys. Rev. Lett. 111, 032501 (2013). 
[72] J. E. Lynn, I. Tews, J. Carlson, S. Gandolfi, A. Gezerlis, K. E. Schmidt, and A. Schwenk, Phys. Rev. Lett. 116, 062501 (2016).

[73] A. Mutschler, A. Lemasson, O. Sorlin, D. Bazin, C. Borcea, R. Borcea, Z. Dombrádi, J.-P. Ebran, A. Gade, H. Iwasaki et al., Nat. Phys. 13, 152 (2017).

[74] T. Otsuka, T. Suzuki, R. Fujimoto, H. Grawe, and Y. Akaishi, Phys. Rev. Lett. 95, 232502 (2005).

[75] W. H. Long, H. Sagawa, N. V. Giai, and J. Meng, Phys. Rev. C 76, 034314 (2007).

[76] A. Arima, M. Harvey, and K. Shimizu, Phys. Lett. B 30, 517 (1969).
[77] K. T. Hecht and A. Adler, Nucl. Phys. A 137, 129 (1969).

[78] J. N. Ginocchio, Phys. Rev. Lett. 78, 436 (1997).

[79] H. Liang, S. Shen, P. Zhao, and J. Meng, Phys. Rev. C 87, 014334 (2013).

[80] S. Shen, H. Liang, P. Zhao, S. Zhang, and J. Meng, Phys. Rev. C 88, 024311 (2013).

[81] H. Liang, J. Meng, and S.-G. Zhou, Phys. Rep. 570, 1 (2015).

[82] X.-L. Ren, K.-W. Li, L.-S. Geng, B. Long, P. Ring, and J. Meng, Chin. Phys. C 42, 014103 (2018).

[83] K.-W. Li, X.-L. Ren, L.-S. Geng, and B.-W. Long, Chin. Phys. C 42, 014105 (2018). 